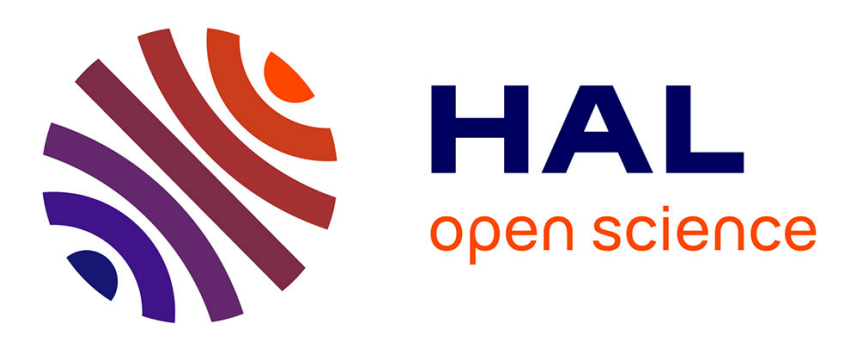

\title{
Etude expérimentale du bruit de fond des amplificateurs et transistors par une méthode de mesure directe
}

\author{
G. Blasquez, J. Borreil
}

\section{To cite this version:}

G. Blasquez, J. Borreil. Etude expérimentale du bruit de fond des amplificateurs et transistors par une méthode de mesure directe. Revue de Physique Appliquée, 1982, 17 (12), pp.793-799. 10.1051/rphysap:019820017012079300 . jpa-00245059

\section{HAL Id: jpa-00245059 https://hal.science/jpa-00245059}

Submitted on 1 Jan 1982

HAL is a multi-disciplinary open access archive for the deposit and dissemination of scientific research documents, whether they are published or not. The documents may come from teaching and research institutions in France or abroad, or from public or private research centers.
L'archive ouverte pluridisciplinaire HAL, est destinée au dépôt et à la diffusion de documents scientifiques de niveau recherche, publiés ou non, émanant des établissements d'enseignement et de recherche français ou étrangers, des laboratoires publics ou privés. 
Classification

Physics Abstracts

$06.30-07.50$

\title{
Etude expérimentale du bruit de fond des amplificateurs et transistors par une méthode de mesure directe
}

\author{
G. Blasquez et J. Borreil \\ Laboratoire d'Automatique et d'Analyse des Systèmes du C.N.R.S., \\ 7, avenue du Colonel Roche, 31400 Toulouse, France
}

(Reçu le 29 mars 1982, révisé le 23 juillet 1982, accepté le 25 août 1982)

\begin{abstract}
Résumé. - La nouvelle méthode de mesure du facteur de bruit des transistors et amplificateurs proposée dans la Revue de Physique Appliquée 15(1980) 1007, a été rediscutée et essayée. Il a été montré que le circuit de base employé dans cette méthode se comporte comme un système à boucle de contre-réaction unitaire et que le gain en boucle ouverte $G_{\mathrm{A}}(f)$ est égal à celui des montages classiques de mesure du facteur de bruit. Il a été précisé que ce circuit permet non seulement les mesures de facteur de bruit mais aussi celles des générateurs équivalents de bruit situés à l'entrée des dispositifs à caractériser. Le calcul des limitations a indiqué que les valeurs admissibles de $G_{\mathrm{A}}(f)$ et la bande des fréquences où les résultats sont significatifs, sont définies par la condition $G_{\mathrm{A}}(f) \geqslant 3$. Les valeurs permises de la résistance de source sont celles qui sont inférieures à l'inverse du module de l'admittance de transfert inverse $y_{12}$. Les essais ont confirmé que cette nouvelle méthode est plus simple à mettre en œuvre, plus précise et plus rapide que les méthodes classiques.
\end{abstract}

\begin{abstract}
The new method proposed in the Revue de Physique Appliquée 15 (1980) 1007, for noise figure measurements in amplifiers and transistors, was modelled and tested. It was shown that the fundamental circuit of this method has the behaviour of a unity feedback system and an open loop gain $G_{\mathrm{A}}(f)$ equal to those found in classical arrangements of noise figure measurements. It was demonstrated that this circuit allows not only noise figure measurements, but also those of the equivalent input noise generators. A calculation of the limitations indicated that the allowed values for $G_{\mathrm{A}}(f)$ and the bandwidth where the results are significant, are defined by the condition $G_{\mathrm{A}}(f) \geqslant 3$. The permitted values of the source resistance are lower than those of the inverse of the reverse transfer admittance $y_{12}$. Experiments confirmed that this method is simpler to use, more accurate and faster than classical methods.
\end{abstract}

1. Introduction. - Dans un article récent [1], il a été montré qu'en connectant la résistance de source entre l'entrée et la sortie d'un quadripôle amplificateur, il était théoriquement possible de déterminer le facteur de bruit du montage d'une manière simple, rapide et précise. Etant donné l'intérêt pratique de cette méthode pour la caractérisation des transistors et des circuits intégrés, l'étude a été poursuivie par des essais de mise en application. Parallèlement, comme l'approche utilisée dans [1] ne permettait pas de voir clairement les ressemblances et les différences de cette méthode par rapport à celles utilisées jusqu'à présent, l'analyse du fonctionnement du montage a été reprise à partir d'un schéma de principe simplifié en utilisant le formalisme des systèmes asservis linéaires.

Les résultats obtenus sont exposés dans ce qui suit. $\mathrm{Au}$ plan théorique l'approche "système " se révèle fructueuse. En particulier, elle permet de préciser les limitations du montage. Au plan expérimental la validité de la méthode proposée dans [1] est démontrée par de nombreux exemples.

L'article est divisé en deux parties principales. Les principes sont réexaminés dans la première. Les résultats expérimentaux sont présentés et analysés dans la seconde.

2. Modélisation et limitations intrinsèques de la méthode directe. - D'une manière classique, pour caractériser en bruit de fond un quadripôle $Q$, dans les domaines des basses et moyennes fréquences, on constitue un étage amplificateur en chargęant $Q$ par une admittance $Y_{L}$ et en l'attaquant par une résistance $R_{\mathrm{S}}$. Le montage correspondant et son circuit équivalent en bruit de fond sont donnés sur la figure 1 où $E_{n Q}$ et $I_{n Q}$ sont les générateurs de bruit de Q et $E_{n S}$ est celui de $R_{\mathrm{S}}$. Par simplicité, nous supposons $Y_{\mathrm{L}}$ non bruyant. 


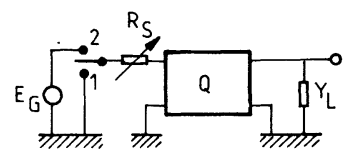

a )

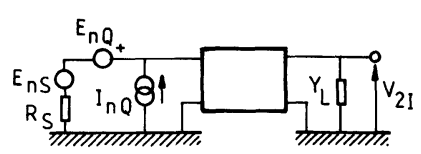

b )

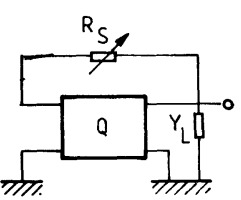

a )

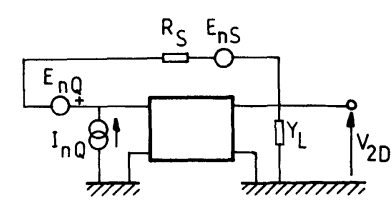

b)
Fig. 1. - a) Montage de principe pour la mesure des paramètres de bruit d'un quadripôle $\mathrm{Q}$. $b$ ) Circuit équivalent en bruit de fond quand le bruit de $Y_{\mathrm{L}}$ est négligé.

[a) Fundamental circuit arrangement for the measure of noise parameters in the twoport $\mathrm{Q}$. b) Noise equivalent circuit when the noise generator of $Y_{\mathrm{L}}$ is neglected.]

Par définition [1], le facteur de bruit du montage s'écrit :

$$
F=\frac{\left\langle\left|E_{n \mathrm{~S}}+E_{n \mathrm{Q}}+R_{\mathrm{S}} I_{n \mathrm{Q}}\right|^{2}\right\rangle}{\left\langle\mid E_{n \mathrm{~S}}^{2}\right\rangle}
$$

où $\left\langle\left|E_{n \mathrm{~S}}\right|^{2}\right\rangle=4 k T_{0} R_{\mathrm{S}} \Delta f, k$ est la constante de Boltzmann, $T_{0}=290 \mathrm{~K}, \Delta$ fun intervalle de fréquences élémentaire. La valeur de $R_{\mathrm{S}}$ étant par hypothèse connue, pour avoir $F$, il faut déterminer le numérateur de (1). D'une manière générale cela s'effectue à partir de la mesure de $\left\langle\left|V_{2 I}\right|^{2}\right\rangle$, valeur quadratique moyenne du bruit à la sortie, et de $G_{\mathrm{A}}(f)$, gain en tension du montage. On a, en effet, quand l'interrupteur est en position 1 :

$$
\left\langle\left|E_{n \mathrm{~S}}+E_{n \mathrm{Q}}+R_{\mathrm{S}} I_{n \mathrm{Q}}\right|^{2}\right\rangle=\frac{\left\langle\left|V_{21}\right|^{2}\right\rangle}{\left|G_{\mathrm{A}}(f)\right|^{2}} .
$$

La valeur théorique de $G_{\mathrm{A}}(f)$ est donnée par :

$$
G_{\mathrm{A}}(f)=\frac{y_{21}}{y_{22}+y_{\mathrm{L}}+R_{\mathrm{S}}\left(\Delta y+y_{11} y_{\mathrm{L}}\right)}
$$

où les paramètres $y_{i j}$ sont les paramètres admittance du quadripôle $Q$ et

$$
\Delta y=y_{11} y_{22}-y_{12} y_{21} \text {. }
$$

Sa détermination s'effectue en injectant un signal $E_{\mathrm{G}}$ à l'entrée du montage (iaterrupteur en position 2) et en mesurant le signal à la sortie.

Dans la méthode directe proposée dans [1], la structure du montage est celle représentée sur la figure 2. La tension de bruit à la sortie est égale à [1] :

$$
\begin{aligned}
& \text { où } \quad V_{2 \mathrm{D}}=k_{1} k_{2}\left(E_{n \mathrm{~S}}+E_{n \mathrm{Q}}+k_{2}^{-1} I_{n \mathrm{Q}}\right) \\
& k_{1} k_{2}=-\frac{y_{11}+y_{21}}{y_{11}+y_{12}+y_{21}+y_{22}+y_{\mathrm{L}}+R_{\mathrm{S}}\left(\Delta y+y_{11} y_{\mathrm{L}}\right)}
\end{aligned}
$$

$$
k_{2}=-\frac{y_{11}+y_{21}}{1-R_{\mathrm{S}} y_{21}} \text {. }
$$

Fig. 2. - a) Montage proposé dans [1] pour la mesure directe des paramètres de bruit d'un quadripôle $Q$. b) Circuit équivalent en bruit de fond.

[a) Arrangement given in [1] for the direct measurement of noise parameters in the twoport $Q$. b) Noise equivalent circuit.]

La méthode est applicable si [1] :

$$
k_{2}^{-1} \approx R_{\mathrm{S}}
$$

c'est-à-dire pour :

$$
\begin{gathered}
R_{\mathrm{S}} \operatorname{Re}\left(y_{21}\right) \gg 1 \\
\left|y_{21}+y_{11}\right| \approx y_{21} \\
\frac{\operatorname{Im}\left(y_{21}+y_{11}\right)}{\operatorname{Re}\left(y_{21}+y_{11}\right)} \approx \frac{\operatorname{Im}\left(y_{21}\right)}{\operatorname{Re}\left(y_{21}\right)} .
\end{gathered}
$$

Dans ces expressions $\operatorname{Re}($ ) et $\operatorname{Im}(\quad)$ désignent respectivement la partie réelle et imaginaire des quantités entre parenthèses.

Le facteur $k_{1} k_{2}$ peut se mettre sous la forme :

où

$$
-k_{1} k_{2}=\frac{G(f)}{1+G(f)}
$$

$$
G(f)=\frac{y_{11}+y_{21}}{y_{12}+y_{22}+y_{\mathrm{L}}+R_{\mathrm{S}}\left(\Delta y+y_{11} y_{\mathrm{L}}\right)} .
$$

Quand (7) à (10) sont vérifiées il est facile de montrer que :

$$
G(f) \approx G_{\mathrm{A}}(f) .
$$

Il résulte de (13), (11) et (7) que le circuit de la figure 2 se comporte comme le système asservi à boucle de contre-réaction de gain unité représenté sur la figure 3.

Pour $G_{\mathrm{A}}(f)$ quelconque, la tension de bruit à la sortie est égale à :

$$
V_{2 \mathrm{D}}=-\frac{G_{\mathrm{A}}(f)}{1+G_{\mathrm{A}}(f)}\left(E_{n \mathrm{~S}}+E_{n \mathrm{Q}}+R_{\mathrm{S}} I_{n \mathrm{Q}}\right) \text {. }
$$

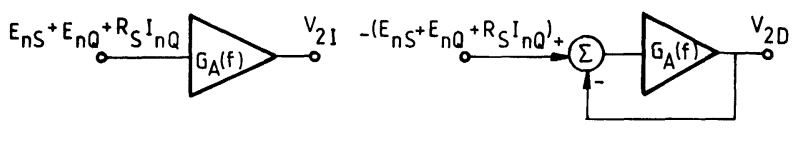

1)

2)

Fig. 3. - Diagrammes synoptiques des circuits des figures 1 et 2 .

[Block diagrams of circuits in figures 1 and 2.] 
Pour $\left|G_{\mathrm{A}}\right| \gg 1, V_{2 \mathrm{D}}$ se réduit à :

et donc :

$$
V_{2 \mathrm{D}}=-\left(E_{n \mathrm{~S}}+E_{n \mathrm{Q}}+R_{\mathrm{S}} I_{n \mathrm{Q}}\right)
$$

$$
F=\frac{\left\langle\left|V_{2 \mathrm{D}}\right|^{2}\right\rangle}{\left\langle\left|E_{n \mathrm{~S}}\right|^{2}\right\rangle}
$$

Puisque $\left\langle\left|E_{n \mathrm{~S}}\right|^{2}\right\rangle$ est connu, pour déterminer $F$, il suffit de mesurer $\left\langle\left|V_{2 D}\right|^{2}\right\rangle$.

Dans les domaines des basses et moyennes fréquences, la connaissance de $\left\langle\left|E_{n Q}\right|^{2}\right\rangle$ et de $\left\langle\left|I_{n Q}\right|^{2}\right\rangle$ est plus utile que celle de $F$. En choisissant $R_{\mathrm{S}}=0$, on obtient :

$$
\left.\left\langle\left|E_{n Q}\right|^{2}\right\rangle \approx\left\langle\left|V_{2 \mathrm{D}}\right|^{2}\right\rangle\right|_{R_{\mathrm{S}}=0}
$$

et pour $R_{\mathrm{S}} \gg R_{\mathrm{so}}$ tel que

on a :

$$
R_{\text {SO }}=\left(\left\langle\left|E_{n Q}{ }^{2}\right|\right\rangle /\left\langle\left|I_{n Q}\right|^{2}\right\rangle\right)^{1 / 2}
$$

$$
\left\langle\left|I_{n \mathrm{Q}}\right|^{2}\right\rangle \approx R_{\mathrm{S}}^{-2}\left[\left\langle\left|V_{2 \mathrm{D}}\right|^{2}\right\rangle-\left\langle\left|E_{n \mathrm{~S}}\right|^{2}\right\rangle\right] \text {. }
$$

Le circuit de la figure 2 permet donc de caractériser d'une manière simple et directe l'ensemble des paramètres de bruit de tout quadripôle amplificateur.

L'intérêt essentiel de ce circuit est d'éviter les mesures de $G_{\mathrm{A}}(f)$. En pratique, aux fréquences élevées, $G_{\mathrm{A}}(f)$ décroît. Par suite, la condition $\left|G_{\mathrm{A}}(f)\right| \gg 1$ n'est plus réalisée partout. Précisons alors la bande des fréquences dans laquelle les résultats obtenus sont acceptables.

Pour cela, admettons que $G_{\mathrm{A}}(f)$ est défini par :

$$
G_{\mathrm{A}}(f)=\frac{G_{\mathrm{A}}(0)}{1+j f / f_{\mathrm{A}}}
$$

avec

$$
\left|G_{\mathrm{A}}(f)\right|=1 \text { pour } f=f_{\mathrm{M}}=G_{\mathrm{A}}(0) f_{\mathrm{A}}
$$

et que par ailleurs $G_{\mathrm{A}}(f)$ peut prendre une valeur quelconque. En première approximation, le gain est constant jusqu'à $f=f_{\mathrm{A}}$ dans le circuit classique et jusqu'à $1+G_{\mathrm{A}}(0) f_{\mathrm{A}}$ dans le circuit de la figure 2 . La connaissance de $G_{\mathrm{A}}(0)$ et la mesure du bruit à la sortie permettent la détermination simple du numérateur de (1) jusqu'à, $f=f_{\mathrm{A}}$ dans le premier cas et jusqu'à $f=f_{\mathrm{A}}\left[1+G_{\mathrm{A}}(0)\right]$ dans le deuxième. Cet avantage du circuit de la figure 2 peut être conservé jusqu'à des fréquences supérieures si une erreur systématique de mesure est acceptée. En effet, désignons par $\left\langle|V(f)|^{2}\right\rangle$ le numérateur de (1). L'expression (14) donne :

$$
V(f)=\frac{1+G_{\mathrm{A}}(f)}{G_{\mathrm{A}}(f)} V_{2 \mathrm{D}}(f) .
$$

Posons :

$$
\hat{V}(f)=\frac{1+G_{\mathrm{A}}(0)}{G_{\mathrm{A}}(0)} V_{2 \mathrm{D}}(f)
$$

et admettons que $\hat{V}(f)$ soit l'estimateur de $V(f)$. Ceci revient à négliger les variations de $G_{\mathrm{A}}(f)$. Définissons l'erreur commise par :

$$
\varepsilon(f)=\frac{\left\langle|V|^{2}\right\rangle-\left\langle|\hat{V}|^{2}\right\rangle}{\left\langle|V|^{2}\right\rangle} .
$$

Pour $\varepsilon(f)$ petit on montre sans difficultés que :

$$
\varepsilon(f)=\left\{\frac{f}{\left[1+G_{\mathrm{A}}(0)\right] f_{\mathrm{A}}}\right\}^{2} .
$$

Si nous acceptons une erreur maximale de $10 \%$, l'expression (23) indique que $\hat{V}(f)$ est un bon estimateur de $V(f)$ jusqu'à $f \leqslant \frac{f_{M}}{3}$ [pour simplifier il a été supposé ici que $\left.1+G_{\mathrm{A}}(0) \approx G_{\mathrm{A}}(0)\right]$. Etant donné que pour un amplificateur la bande de fréquences qui présente de l'intérêt est définie par $f<f_{\mathrm{M}}$, on voit que le circuit de la figure 2 doit permettre la détermination des caractéristiques de bruit dans la quasi-totalité de cette bande.

En complément de ce qui a été exposé dans [1] sur les limitations de la méthode de mesure directe, il est intéressant d'examiner d'une manière plus approfondie la condition (8) sur les plus petites valeurs admissibles de $R_{\mathrm{s}}$. Pour $\boldsymbol{R}_{\mathrm{S}}$ faible, l'expression (6) indique que : $\left|k_{2}^{-1}\right|<R_{\mathrm{S}}$ et donc que $k_{2}^{-1} I_{n \mathrm{Q}}<R_{\mathrm{s}} I_{n \mathrm{Q}}$. En théorie il peut en résulter une erreur sur l'estimation de $V(f)$. En réalité, pour les transistors et amplificateurs opérationnels si $R_{\mathrm{s}}$ est faible, $V(f) \approx E_{n \mathrm{~s}}+E_{n \mathrm{Q}}$, c'est-à-dire que la contribution du terme $R_{\mathrm{S}} I_{\mathrm{nQ}}$ est négligeable. Alors $k_{2}^{-1} I_{n \mathrm{Q}} \neq R_{\mathrm{S}} I_{n \mathrm{Q}}$ n'a aucune influence. Une analyse plus détaillée de ce problème montre que la condition (8) peut être ignorée tant que $R_{\mathrm{S}} \ll R_{\text {So }}$ et donc si $R_{\text {so }} \gg\left[\operatorname{Re}\left(y_{21}\right)\right]^{-1}$.

Enfin, remarquons que dans tout quadripôle, il existe un effet de contre-réaction interne caractérisé par le paramètre $y_{12}$. Pour que le montage de la figure 2 fonctionne correctement, il faut que l'essentiel de la contre-réaction soit effectuée par $R_{\mathrm{S}}$. En conséquence, la limite supérieure des valeurs permises pour $\boldsymbol{R}_{\mathrm{S}}$ est :

$$
R_{\mathrm{S}} \ll\left|y_{12}\right|^{-1} \text {. }
$$

3. Etude expérimentale. - L'étude a porté sur des amplificateurs opérationnels, des transistors bipôlaires et des transistors à effet de champ.

Les paramètres caractérisés ont été les générateurs équivalents $E_{n \mathrm{Q}}$ et $I_{n \mathrm{Q}}$. L'analyse du bruit a été effectuée dans une bande de fréquences allant de $10 \mathrm{~Hz}$ à $100 \mathrm{kHz}$ avec le système numérique décrit dans [2]. Sa précision est de l'ordre de $10 \%$.

Pour simplifier la procédure expérimentale, et pour vérifier que l'effet des variations de $G_{\mathrm{A}}(f)$ sur la méthode directe est translaté vers les hautes fréquences, les résultats des mesures données par la méthode classique ont été exploités en prenant pour $G_{\mathrm{A}}(f)$ la valeur $G_{\mathrm{A}}(0)$. 
3.1 ETUDE DES AMPLIFICATEURS INTÉGRÉS. - Les amplificateurs testés étaient du type 741. Leurs spécifications étaient les suivantes : gain en boucle ouverte $10^{5}$, fréquences de cassures $f_{1}=10 \mathrm{~Hz}$ et $f_{2}=1 \mathrm{MHz}$, bruit en tension à l'entrée $17 \mathrm{nV} / \sqrt{\mathrm{Hz}}$ à $100 \mathrm{kHz}$, bruit en courant à l'entrée $5,5 \times 10^{-13} \mathrm{~A} / \sqrt{\mathrm{Hz}}$ à $100 \mathrm{kHz}$.

Les montages utilisés pour déterminer $E_{n \mathrm{O}}$ sont reproduits sur la figure 4. Dans le cas de la méthode classique, l'amplificateur avait en basse fréquence un gain $G_{\mathrm{A}}(0) \approx 10$. Les résistances utilisées avaient des faibles valeurs pour que leurs contributions en bruit puissent être négligées. Dans l'autre cas, l'amplificateur était connecté en suiveur de tension. La propriété essentielle de ce montage est que la tension de sortie est égale à la tension d'entrée. Il en résulte que le bruit à la sortie est égal au bruit à l'entrée puisque les seules sources de signal présentes dans le circuit sont les générateurs de bruit. Il est intéressant de remarquer que le montage a la structure de la figure 3. Les résultats sont donnés sur la figure 5 . Il y a un bon accord jusqu'à $20 \mathrm{kHz}$. En particulier la valeur observée est approximativement celle spécifiée par le fabricant. Au-delà la courbe donnée par la méthode indirecte décroît à cause de l'hypothèse

$$
G_{\mathrm{A}}(f)=G_{\mathrm{A}}(0)=\text { Cte quel que soit } f .
$$

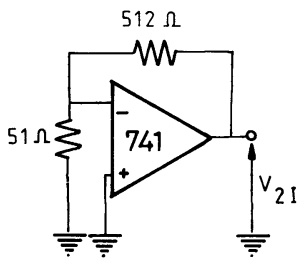

a)

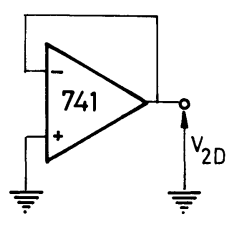

b)
Fig. 4. - Montages pour la mesure du générateur de bruit en tension placé à l'entrée des amplificateurs intégrés : a) Méthode directe. $b$ ) Méthode classique.

[Measurement arrangements.]

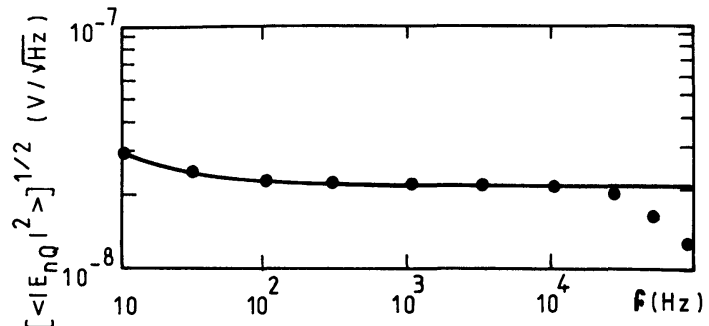

Fig. 5. - Valeur efficace du générateur de bruit en tension placé à l'entrée d'un amplificateur opérationnel du type 741 . Méthode directe _

[R.M.S. value of the equivalent input noise generator of an operational amplifier, model 741 : direct method classical method assuming $G_{\mathrm{A}}(f)$ constant 00.$]$
On voit clairement que la décroissance de $G_{\mathrm{A}}(f)$ n'a pas d'influence sur les résultats donnés par la méthode de mesure directe.

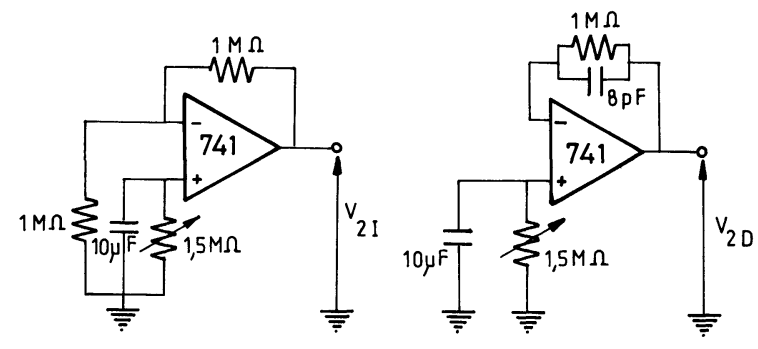

a )

b )

Fig. 6. - Montage de mesure du générateur de bruit en courant placé à l'entrée des amplificateurs intégrés : $a$ ) Méthode classique. $b$ ) Méthode directe.

[Measurement arrangements of the equivalent input current noise generator in integrated amplifiers : $a$ ) Classical method. b) Direct method.]

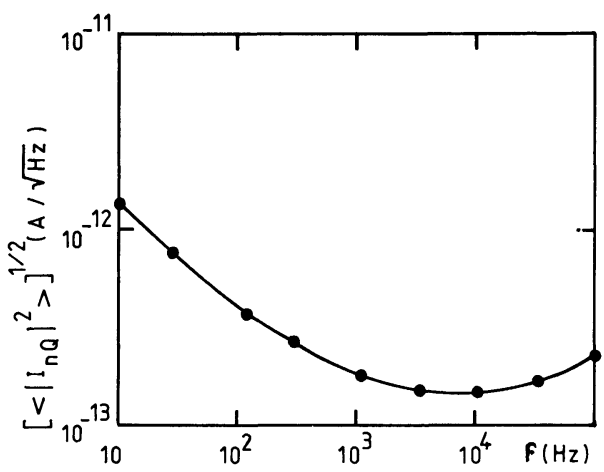

Fig. 7. - Valeur efficace du générateur de bruit en courant placé à l'entrée d'un amplificateur du type 741. Méthode directe —., Méthode classique 000.

[R.M.S. value of the equivalent input current noise generator in on operational amplifier, model 741 : direct method classical method assuming $G_{\mathrm{A}}(f)$ constant 000 .]

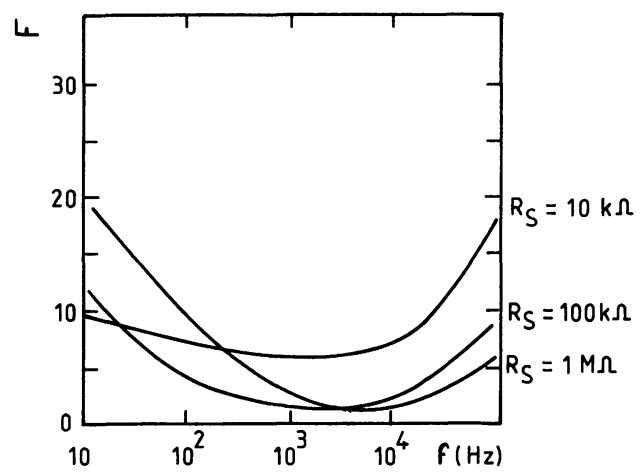

Fig. 8. - Facteur de bruit d'un amplificateur type 741 en fonction de la fréquence pour des résistances de source $R_{\mathrm{s}}$ égales à $10 \mathrm{k} \Omega, 100 \mathrm{k} \Omega$ et $1 \mathrm{M} \Omega$. Le montage utilisé est celui de la figure $6(b)$.

[Noise figure of a 741 operational amplifier for source resistances equal to $10 \mathrm{k} \Omega, 100 \mathrm{k} \Omega$ and $1 \mathrm{M} \Omega$. The experimental arrangement is similar to figure $6(b)$.] 
La caractérisation du générateur $I_{n \mathrm{Q}}$ a été effectuée avec les circuits de la figure 6. Le réseau RC placé sur l'entrée positive a deux rôles. En régime dynamique c'est un court-circuit. En régime statique, il sert à équilibrer les tensions continues induites par les courants des étages d'entrée. Il est intéressant de noter que le montage de mesure directe est celui d'un électromètre et donc que la tension de sortie est égale à $R_{\mathrm{S}} I_{\mathrm{nQ}}$. La capacité $C_{\mathrm{s}}=8 \mathrm{pF}$ donne la stabilité. Pour les valeurs élevées de $R_{\mathrm{S}}$, l'effet combiné de $R_{\mathrm{S}}$ et de l'impédance d'entrée introduit dans $G_{\mathrm{A}}(f)$ un pôle compris entre $f_{1}$ et $f_{2}$. Le montage se comporte comme un système de $3^{\mathrm{e}}$ ordre instable. Le réseau $R_{\mathrm{S}} / / C_{\mathrm{S}}$ produit dans $G_{\mathrm{A}}(f)$ un zéro. En donnant à $C_{\mathrm{S}}$ une valeur adéquate, l'effet du pôle compris entre $f_{1}$ et $f_{2}$ est compensé et le comportement oscillant du montage est supprimé.

Les résultats obtenus par les deux méthodes sont reproduits sur la figure 7 . Ils sont quasiment identiques.

A titre de complément, le facteur de bruit avait été déterminé pour $R_{\mathrm{S}}=10 \mathrm{k} \Omega, 100 \mathrm{k} \Omega$ et $1 \mathrm{M} \Omega$. Nous voyons sur la figure 8 que la valeur la plus faible de $F$ a été obtenue pour $R_{\mathrm{S}}=100 \mathrm{k} \Omega$. A partir des mesures de $E_{n Q}$ et de $I_{n Q}$ nous calculons que le facteur de bruit minimum doit être observé pour $R_{\text {so }} \approx 120 \mathrm{k} \Omega$. Nous constatons que les résultats des mesures de $E_{n Q}, I_{n Q}$ et $F$ sont cohérents.

\subsection{ETUde DES TRANSISTORS A EFFET DE CHAMP. -} L'étude a été effectuée sur des transistors intégrés de la filière technologique BIFET. La valeur de la transconductance $G_{\mathrm{m}}$ à saturation était voisine de $3,5 \times 10^{-4} \Omega^{-1}$. Les montages qui ont été réalisés sont dessinés sur la figure 9. Pour minimiser les contributions en bruit des circuits de polarisation, il a été utilisé des résistances à couches métalliques et des batteries $\mathrm{CdNi}$.

Nous n'avons caractérisé que le générateur $E_{n \mathrm{O}}$ parce qu'en basses et moyennes fréquences le bruit

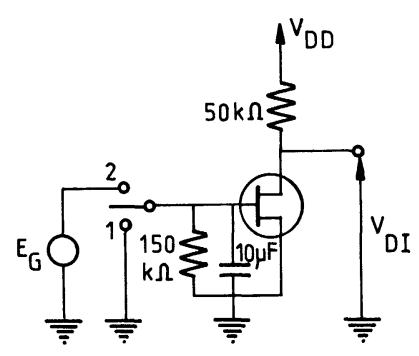

a)

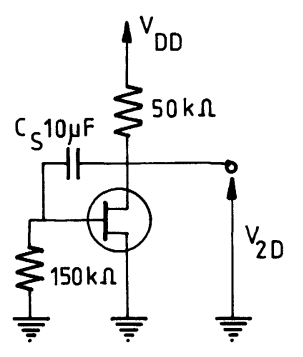

b )
Fig. 9. - Montages de mesure du générateur de bruit en tension placé à l'entrée des transistors à effet de champ : $a$ ) Méthode classique. $b$ ) Méthode directe.

[Measurement arrangement of the equivalent input voltage noise in field effect transistors. a) Classical method. b) direct method.] prédominant est le bruit en tension. Dans le montage de mesure directe, le court-circuit entrée-sortie a été fait en régime dynamique avec une capacité de forte valeur au tantale. Dans tous les cas, le circuit contreréactionné a eu un comportement stable. La figure 10 montre les résuiltats obtenus. L'accord entre les deux méthodes de mesure est satisfaisant jusqu'à $20 \mathrm{kHz}$. Dans la bande de fréquences où le bruit a un spectre blanc on voit que la valeur mesurée est égale à : $\left[(2 / 3) 4 k T_{0} / G_{m}\right]^{1 / 2}$.

Notons que cette valeur est celle du bruit thermique généré dans le canal du transistor et qu'en aucun cas, le bruit mesuré ne peut lui être inférieur. La décroissance du bruit observée au-delà de $20 \mathrm{kHz}$ est donc un artefact. Elle résulte d'un effet de filtrage du réseau de sortie RC constitué par la résistance de charge et la capacité d'entrée du préamplificateur de la chaîne de mesure. Remarquons que cet effet n'apparaît pas sur les résultats donnés par la méthode directe. Cette différence est due au fait que l'impédance de sortie du circuit de la figure 2 est $1+G_{\mathrm{A}}(f)$ fois plus petite que celle du circuit de la figure 1 .

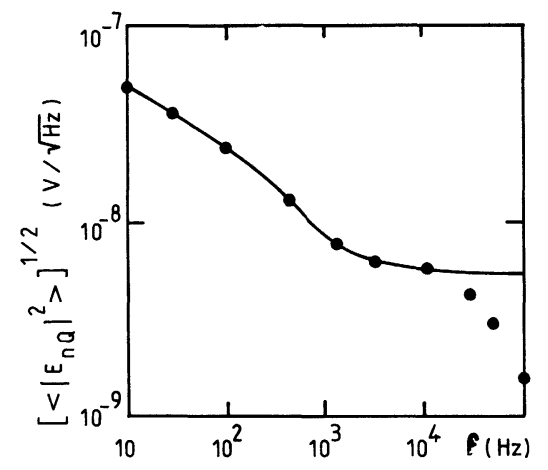

Fig. 10. - Valeur efficace du générateur de bruit en tension placé à l'entrée d'un transistor à effet de champ (Technologie BIFET) : Méthode directe —, Méthode classique $\bullet \bullet \bullet$.

[R.M.S. value of the equivalent input voltage noise generator in a field effect transistor (BIFET technology) : direct method - classical method assuming $G_{\mathrm{A}}(f)$ constant ๑).]

3.3 ETUDE DES TRANSISTORS BIPÔLAIRES. - L'étude du générateur $E_{n Q}$ a été effectuée sur des 2 N 2484 pour des courants collecteurs $I_{\mathrm{c}}$ compris entre $1 \mu \mathrm{A}$ et $1 \mathrm{~mA}$ et pour des différences de potentiel collecteur émetteur de l'ordre de 5 à $6 \mathrm{~V}$. La figure 11 représente les montages mis en ouvre. Le circuit contre-réactionné n'a pas montré de comportements oscillants. La figure 12 donne les résultats obtenus à bas niveau de polarisation. On voit que l'accord est excellent jusqu'à $10 \mathrm{kHz}$. Dans la gamme des fréquences où le spectre du bruit est blanc, la valeur mesurée est égale à $\left[2 k T r_{\mathrm{e}}\right]^{1 / 2}$ avec $r_{\mathrm{e}}=k T /\left(q I_{\mathrm{c}}\right)$. En aucun cas le 
bruit mesuré ne peut lui être inférieur [3]. La cause des divergences observables sur la figure 12 pour $f>10 \mathrm{kHz}$ est la même que dans le cas précédent.

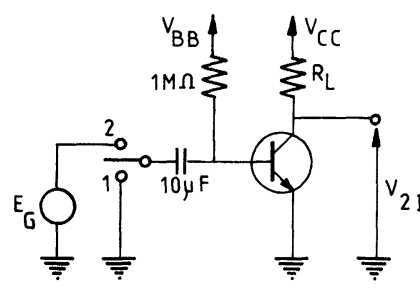

a)

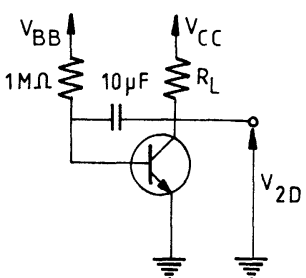

b)
Fig. 11. - Montages de mesure du générateur de bruit en tension placé à l'entrée des transistors bipôlaires : $a$ ) Méthode classique. $b$ ) Méthode directe.

[Measurement Arrangement of the equivalent input voltage noise generator in bipolar transistors : a) Classical method, b) Direct method.]

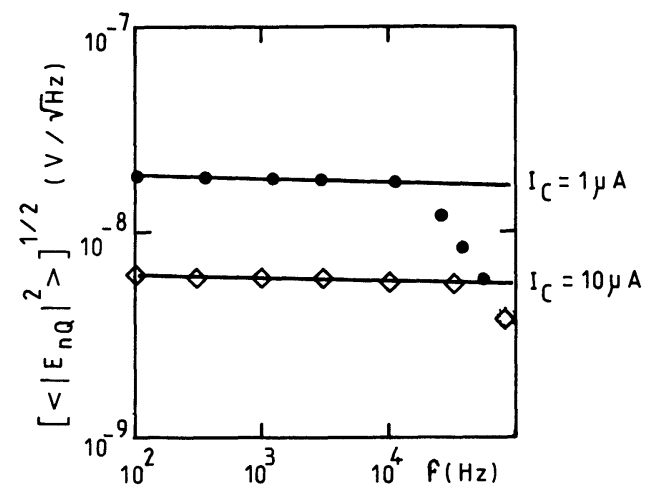

Fig. 12. - Valeur efficace du générateur de bruit en tension placé à l'entrée d'un transistor bipôlaire NPN 2484 : Méthode directe —, Méthode indirecte $\bullet, \diamond$.

[R.M.S. value of the equivalent input voltage noise generator of an 2484 NPN bipolar transistor : direct method classical method $\bullet, \diamond$.]

L'étude du générateur $I_{n Q}$ a été faite sur des transistors intégrés fabriqués par Motorola et qui portent la référence 4759. Les circuits de mesure sont indiqués sur la figure 13. Le comportement du circuit bouclé a été stable dans tous les cas. Des exemples de résultats sont reportés sur la figure 14. L'accord entre les deux méthodes est bon presque partout et les valeurs observées dans les bandes de fréquences où le bruit est blanc sont celles prévues théoriquement [3]. La décroissance donnée par la méthode indirecte pour $f>10 \mathrm{kHz}$ à $I_{\mathrm{c}}=300 \mu \mathrm{A}$ est due à l'effet Miller.

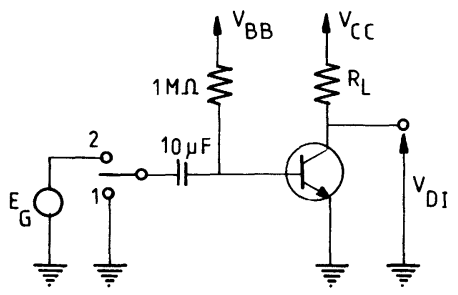

a )

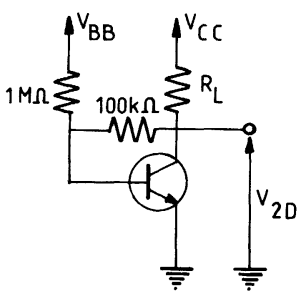

b)
Fig. 13. - Montages de mesure du générateur de bruit en courant placé à l'entrée des transistors bipôlaires : a) Méthode classique, $b$ ) Méthode directe.

[Measurement arrangement of the equivalent input current noise generator in bipolar transistors : a) Classical method, b) Direct method.]

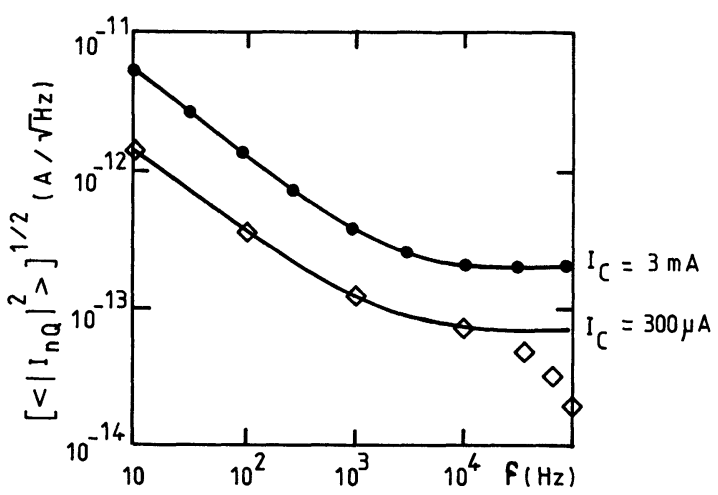

Fig. 14. - Valeur efficace du générateur de bruit en courant d'un transistor bipolaire Motorola 4759. Méthode directe $\longrightarrow$, Méthode classique $\bullet, \diamond$.

[R.M.S. value of the equivalent input current noise generator in a Motorola transistor, model 4759 : direct method , classical method $\bullet, \diamond$.]

4. Conclusion. - Dans une étude récente [1], nous avions montré qu'en plaçant une résistance $R_{\mathrm{S}}$ entre l'entrée et la sortie d'un quadripôle amplificateur il était théoriquement possible de déterminer son facteur de bruit d'une manière directe.

Dans la première partie de cet article, l'analyse du fonctionnement du montage a été reprise à l'aide du formalisme des systèmes asservis linéaires. Il a été établi que ce montage se comporte comme un système dont le gain en boucle ouverte $G_{\mathrm{A}}(f)$ est égal à celui des circuits classiques de mesure du facteur de bruit et dont le gain de la chaîne de contre-réaction est égal à l'unité. A partir de ces propriétés, il a été mis en évidence que pour obtenir des mesures correctes, il faut se limiter au domaine des fréquences pour lequel $G_{\mathrm{A}}(f) \geqslant 3$ et aux valeurs des résistances $R_{\mathrm{S}}$ telles que $R_{\mathrm{S}}<\left|y_{12}\right|^{-1}$. Enfin, il a été précisé que le montage peut servir non seulement à la mesure du facteur de bruit mais aussi à celle des générateurs équivalents placés à l'entrée du quadripôle. 
Dans la deuxième partie de l'article, les résultats des essais de mise en application de la méthode ont été présentés et comparés à ceux donnés par les méthodes de mesures classiques. On a vu qu'en général la structure des circuits à réaliser pour la mesure directe est simple.

Leur conception ne pose pas de problèmes particuliers pour les transistors bipôlaires et pour les transistors à effet de champ. Par contre, des problèmes de stabilité se sont manifestés avec les amplificateurs intégrés. Dans ce cas, il a fallu mettre en ouvre les techniques classiques de compensation des circuits asservis en prenant garde de ne pas introduire des sources de bruit parasites.

En ce qui concerne les chaînes de mesure, la méthode directe a demandé un préamplificateur très faiblement bruyant parce que la valeur quadratique moyenne du bruit en sortie est $\left|G_{\mathrm{A}}(f)\right|^{2}$ fois plus faible que celle délivrée par les montages classiques. Comme prévu dans [1], la détermination des générateurs équivalents et du facteur de bruit par la méthode directe s'est effectuée à partir d'une seule mesure dans un domaine de fréquences étendu. Les résultats obtenus ont été identiques à ceux donnés par les méthodes classiques et conformes aux spécifications des fabricants et aux lois théoriques. L'étude expérimentale confirme donc pour l'essentiel les prévisions théoriques établies dans [1].

Le tableau résume les avantages et les inconvénients de chaque méthode.

Tableau. - Comparaison des avantages et inconvénients des méthodes de mesure classiques des paramètres de bruit des quadripôles et de celle proposée dans [1]. La sensibilité aux variations de $G_{\mathrm{A}}$ est définie par : $S=\mathrm{d}\left(\ln V_{2}\right) / \mathrm{d}\left(\left|G_{\mathrm{A}}(f)\right|\right)$.

Classe des quadripôles concernés

Paramètres à mesurer
Méthode classique

Quelconque

Bruit à la sortie et Gain en tension du montage : $G_{\mathrm{A}}(f)$

Nombre de mesures à effectuer pour cha- $3\left(\mathrm{Si} E_{\mathrm{G}}\right.$ est inconnu) que valeur de la fréquence

Durée des manipulations

Précision des résultats

Complexité du montage de base

Préamplificateur de la chaîne de mesure

Domaine fréquentiel d'application

Gamme des résistances de source admis- Pas de limitation sibles

Problèmes de stabilité

Sensibilité aux variations de $\left|G_{\mathrm{A}}(f)\right|$

Automatisation

Impédance de sortie
Non

\section{Longue}

Pas très élevée

Générateur de signal nécessaire

Faible bruit

Très étendu

1

Complexe

$Z_{0}$
Méthode proposée dans [1]

Amplificateur

Bruit de sortie

1

- Courte

Améliorée, erreurs sur $G_{\mathrm{A}}(f)$ supprimées

Pas de générateur

Très faible bruit

Etendu mais limité par la condition $G_{\mathrm{A}}(f) \geqslant 3$

$R_{\mathrm{S}} \ll\left|y_{12}\right|^{-1}$

Eventuels

$\frac{1}{1+G_{\mathrm{A}}(f)}$

Simple

$\frac{Z_{0}}{1+G_{\mathrm{A}}(f)}$

Bibliographie

[1] Blasquez, G., Revue Phys. Appl. 15 (1980) 1007.

[2] Blasquez, G. and Tosi, R., A High Performance Digital System for noise characterization in electronic devices. Proceedings of the 9th Imeko World Congress, Berlin (West), May 24-28 (1982).

[3] Blasquez, G., Caminade, J. and Van Vliet, K., Solid State Electron. 23 (1980) 423. 